ESMO-Guidelines

\section{Imatinib bei GIST}

Für die Erstlinientherapie von Patienten mit c-kit-positiven, nicht resezierbaren und/oder metastasierten gastrointestinalen Stromatumoren (GIST) empfiehlt die ESMO In ihren Guidelines von 2010 Imatinib (Glivec $\left.{ }^{\circledR}\right) 400$ mg/Tag.

Mehr als $90 \%$ der Patienten mit einer c-kit-Mutation im Bereich des Exons 11 profitieren von einer solchen Therapie. Bei etwa $10 \%$ der Erkrankten liegt die Mutation jedoch im Exon 9. Diese Patienten sollten der European Society for
Medical Oncology (ESMO) zufolge $800 \mathrm{mg}$ Imatinib am Tag erhalten. Grundlage für diese Empfehlung sind u.a. die Ergebnisse einer Phase-III-Studie. Durch eine Dosiserhöhung von 400 auf $800 \mathrm{mg}$ verlängert sich das progressions- freie Überleben auf 19 Monate (vs. 6 Monate bei $400 \mathrm{mg} / \mathrm{Tag}$ ) [van Glabbeke MM et al. J Clin Oncol. 2007; 25: Abstract 10004, Gastrointestinal Stromal Tumor MetaAnalysis Group (MetaGIST). J Clin Oncol. 2010; 27(7): 1247-53]. Aufgrund dieser Unterschiede in der Wirksamkeit sollte zudem bei Diagnosestellung eine Mutationsanalyse erfolgen. Auch bei einer Progression unter $400 \mathrm{mg}$ sollte die Dosis laut ESMO auf $800 \mathrm{mg} /$ Tag erhöht werden.

\section{Novartis Oncology}

\title{
S3-Leitlinie
}

\section{Tyrosinkinasehemmer bei metastasiertem Pankreaskarzinom}

\section{In der aktuellen S3-Leitlinie „Exokrines Pankreaskarzinom“ wird für Patienten mit metastasiertem Tumor der Einsatz des Tyrosin- kinasehemmers Erlotinib (Tarceva ${ }^{\circledR}$ ) empfohlen.}

Erlotinib sei die bislang einzige Substanz, die in der Kombination mit Gemcitabin gegenüber der Gemcitabin-Monotherapie einen signifikanten Überlebensvorteil bot, so Frank Kullmann, Weiden,. Zwar lag der Überlebensvorteil für die Kombinationstherapie insgesamt in der Zulassungsstudie nur im Bereich eines halben Monats, bei den Patienten, die unter Erlotinib ein Hautexanthem vom Schweregrad $>2$ entwickelten, verlängerte sich das mediane Überleben durch die Kombination jedoch um über 4 Monate: von 5,9

Kurz notiert

Hypoallergene Docetaxel-Rezeptur

NeoCorp hat in der Formulierung von Docetaxel NC $10 \mathrm{mg} / \mathrm{ml}$ den Polysorbat 80-Gehalt um $85 \%$ reduziert. Damit soll die Häufigkeit der Überempfindlichkeitsreaktionen gesenkt werden. Als Ersatzstoff dient das gut verträgliche Polyethylenglykol (PEG, Macrogol). Bis zu 26\% der Patienten entwickeln unter Monotherapie $\left(100 \mathrm{mg} / \mathrm{m}^{2}\right)$ Überempfindlichkeitsreaktionen. $5 \%$ erleiden schwere Grad-3/4-Reaktionen. Auch Docetaxel enthaltende Kombinationsschemata führen bei bis zu $11 \%$ der Patienten zu Überempfindlichkeitsreaktionen [Hasmüller S et al. Im Focus Onkologie 2008; 11: 70-76]. Diese Reaktionen werden auf den Stabilisator Polysorbat 80 zurückgeführt. Docetaxel $\mathrm{NC}^{\circledast} 10 \mathrm{mg} / \mathrm{ml}$ wird entsprechend den jeweiligen Therapiestandards bei bestimmten Formen von Brustkrebs, Lungentumoren, Hormon-refraktären Prostatakarzinomen, Adenokarzinomen des Magens und bei Kopf-Hals-Tumoren als 1-h-Infusion verabreicht.

NeoCorp

auf 10,5 Monate [Moore MJ et al. J Clin Oncol 2007;25:1960-6]. Den Zusammenhang zwischen Exanthemgrad und Überlebenszeit unter Erlotinib bestätigt eine auf dem ASCO 2010 vorgestellte Studie [Manzano J et al. J Clin Oncol 28:15s, 2010 (suppl; abstr 4094)]. Kullmann empfahl daher mit der Kombination Gemcitabin/Erlotinib zu beginnen und sie fortzuführen, wenn sich ein Hautausschlag entwickelt. Ohne Exanthem sollte diese Therapie kritisch überdacht werden.

Dass auch Capecitabin (Xeloda ${ }^{\varpi}$ als Kombinationspartner denkbar ist, zeigte die PK-0104-Studie [Boeck S et al. J Clin Oncol 2010;28:18s (suppl; abstr LBA4011)]. Hier wurden zwei Therapieregime verglichen: in der First-Line Erlotinib plus Capecitabin, bei Progress Gemcitabin in der Second-Line und die Kombination Erlotinib/ Gemcitabin als Erstlinien-Therapie, gefolgt von der Monotherapie mit Capecitabin nach Progression unter der Kombination. Das Gesamtüberleben und die Zeit bis zum Versagen der Erst- und Zweitlinientherapie zeigten dabei keine signifikanten Unterschiede. af

Quelle: Satellitensymposium anlässlich der 65. Jahrestagung der Deutschen Gesellschaft für Verdauungs- und Stoffwechselkrankheiten am 16.9.2010 in Stuttgart; Veranstalter: Roche Pharma 